Florian Neuberger*, Julian Baranyai, Torben Schmidt, Thorsten Cottre, Bernhard Kaiser, Wolfram Jaegermann and Rolf Schäfer

\title{
From Bulk to Atoms: The Influence of Particle and Cluster Size on the Hydrogen Evolution Reaction
}

https://doi.org/10.1515/zpch-2019-1424

Received March 29, 2019; accepted September 13, 2019

\begin{abstract}
To investigate the influence of particle size in terms of electrocatalysis for the hydrogen evolution reaction (HER), small $\mathrm{Pt}_{n}$ species with $n=1,10,13$ atoms and nanoparticles are deposited onto native titanium dioxide. These species are compared to the bare support as well as to bulk platinum with respect to the catalytic activity. Photoelectron spectroscopy showed Pt4f core-level shifts to higher binding energies with decreasing cluster size. In addition, the various species contribute significant density of states into the valence band gap of $\mathrm{TiO}_{2}$, thereby with larger particle size, the resulting band gap narrows. For nanoparticles, metal-like behaviour was already observed. Electrochemical measurements in $0.1 \mathrm{M} \mathrm{H}_{2} \mathrm{SO}_{4}$ showed the highest overall catalytic activity for bulk platinum and large Pt nanoparticles. A different assertion is obtained when the activities are related to the mass of the catalyst used, indicating that clusters with a size of about ten atoms seem to be most active. In comparison with the results from photoelectron spectroscopy regarding the electronic structure, no clear correlation to the catalytic activity was found. In terms of degradation induced due to the electrochemical treatment, the cluster samples showed no sintering effects, but instead, some detachment took place.
\end{abstract}

Keywords: cluster; electrocatalysis; native $\mathrm{TiO}_{2}$; platinum; water.

\footnotetext{
*Corresponding author: Florian Neuberger, Eduard-Zintl-Institut, Technische Universität Darmstadt, Alarich-Weiss-Straße 8, D-64287 Darmstadt, Germany, e-mail: neuberger@cluster.pc.chemie.tu-darmstadt.de

Julian Baranyai, Torben Schmidt and Rolf Schäfer: Eduard-Zintl-Institut, Technische Universität Darmstadt, Alarich-Weiss-Straße 8, D-64287 Darmstadt, Germany Thorsten Cottre, Bernhard Kaiser and Wolfram Jaegermann: Surface Science Division, Department of Materials Science, Technische Universität Darmstadt, Otto-Berndt-Str. 3, D-64287 Darmstadt, Germany
} 


\section{Introduction}

Since the early 2000s catalysis, using nanoparticles and nanostructured surfaces is of rising interest in research [1-5]. A major goal for a wide variety of researchers is to reduce the amount of energy or material needed to drive the desired reaction. To achieve this highly effective catalysts are necessary. One strategy is to synthesise new bulk compounds which are feasible to catalyse the reaction [6], whereas the use of nanoparticles and clusters of already known materials can offer an alternative approach [7-9]. The smaller a particle, the more sensitive are its properties to changes in particle size. So each change in atom count can influence the overall catalytic activity [10]. Therefore it is conspicuous, that there might be an ideal particle size for a given material and reaction, which offers the best catalytic performance [11]. Regarding the shortage of non-abundant metals [12], this might also be the case for the ideal particle size of platinum particles to catalyse the hydrogen evolution reaction (HER) and therefore to minimise the amount needed to generate hydrogen via electrolysis. A similar effect was already demonstrated e.g. by Weber et al. for the oxygen reduction reaction on indium tin oxide (ITO) [13], as well as for platinum clusters for the photocatalytic HER on CdS nanorods [14]. Here the $\mathrm{Pt}_{46}$-cluster showed a two times higher hydrogen production rate than the larger $\mathrm{Pt}_{68}$-cluster or the smaller $\mathrm{Pt}_{34}$-cluster.

For an efficient setup to produce solar hydrogen, a good photoabsorber material is needed in addition which can provide a high yield and simultaneously delivers enough electric energy to overcome the necessary barrier to split water. One promising absorber for this task are silicon-based multi-junction cells [15]. Regarding the enhancement of such a photovoltaic-electrochemistry (PV-EC) cell setup, band alignment and charge transfer effects between the different junctions and interfaces to the liquid phase must be contemplated [16]. Especially titanium dioxide can be used to create a thin, passivating and conductive interlayer on silicon-based solar cells [17] and is of recent interest for a diversity of photoabsorber materials $[18,19]$. Altough titanium dioxide as catalyst support bears problems concerning the conductivity [20] and the low intrinsic catalytic activity [21-23], it has also been intensively investigated during electrochemical processes [24]. The research of PV-EC with $\mathrm{TiO}_{2}$ as electrocatalytic layer by Lichterman et al. indicated a band shift at the liquid interface by using atmospheric pressure X-ray photoelectron spectroscopy (XPS). They revealed that at a certain potential the pinning of the Fermi level to the defect states of the conductive layer occurred [25]. To improve the electrocatalytic performance of the terminating solid layer a noble metal co-catalyst can be added. In a most recent theoretical investigation by Wang et al. of the size-dependent activity of small platinum clusters at titanium oxide 
it is found that the surface catalytic performance of the HER is highest with $\mathrm{Pt}_{8}$, $\mathrm{Pt}_{13}$ and $\mathrm{Pt}_{19}$ particles compared to smaller species. The opposite tendency was observed for the electron transfer performance. Here the isolated Pt-atom looks to be most effective [26]. Taking both effects into account, Pt-clusters with about thirteen atoms seem to be most active.

Since in photocatalysis both the electron transport and the catalytic activity are of importance, here we want to exclusively study the electrocatalytic properties of Pt-species, i.e. our approach is to focus on the electrochemical section of a multi-junction PV-EC cell. To investigate the influence of particle size on the catalytic activity, a variety of different species were deposited on native titanium dioxide by magnetron sputtering and electrodeposition. Samples from the isolated atom to larger nanoparticles were further analysed by photoelectron spectroscopy and investigated via electrochemical cyclic voltammetry. This paper presents the first promising results and depicts our methodology to investigate the electrocatalytic performance of supported small metal clusters.

\section{Experimental methods}

\subsection{Sample preparation}

As deposition support material titanium sheets (99.93\%, Alfa Aesar) with a thickness of $0.5 \mathrm{~mm}$ and an area of $10 \times 10 \mathrm{~mm}^{2}$ were used. The experimental determination of the native oxide film thickness was done using X-ray photoelectron spectroscopy (XPS). A value of $2.5 \pm 0.6 \mathrm{~nm}$ was found for the thickness of the native titanium dioxide layer. This is in accordance with several thin film studies reported in the literature [27-33]. As a reference material, a $0.25 \mathrm{~mm}$ thick platinum foil (99.995\%, Alfa Aesar) was used. All samples were cleaned for 5 min each with acetone, isopropanol and ethanol in an ultrasonic bath. The solvents were all purchased as analytic grade (Carl Roth).

\subsubsection{Platinum deposition}

The vacuum apparatus used for cluster deposition consists of four differentially pumped vacuum chambers with a base pressure of at least $1 \cdot 10^{-9}$ mbar in the deposition chamber. Clusters are produced in a source chamber with an ONYX I 
Mag II IC magnetron cathode for magnetron sputtering. As a platinum source, a Canadian Maple Leaf with 99.95\% platinum was used. Argon (Westfalen, 99.999\%) was used as sputtering gas. Subsequently, the metal vapour is cooled by a mixture of helium (Westfalen 99.9999\%) and argon while clusters begin to form. Afterwards, the mixture expands through an orifice into high vacuum. A skimmer separates Pt-atoms and clusters from lighter noble gases. Then, complex ion optics consisting of einzel lenses, a quadrupole mass filter (Extrel tri-filter, $440 \mathrm{kHz}$ ) and a quadrupole ion guide (Beam Imaging Solutions, QID-900-H) are used to transfer, filter a given cluster size and separate neutral from charged particles, respectively. Upon leaving the quadrupole ion deflector, the positively charged clusters are deposited on $10 \times 10 \mathrm{~mm}^{2}$ titanium sheet metal with a native layer of oxide. To adjust the impact energy of clusters on the surface, an electric potential was applied to the sample. Therefore, clusters were soft-landed on the support with a kinetic energy of less than $0.5 \mathrm{eV}$ per atom. To ensure homogeneity over the whole sample the beam profile was measured in situ by a $3 \times 3$ array of copper plates which record the incoming cluster current. The cluster coverage for the monodisperse samples (platinum atoms $\mathrm{Pt}_{\text {atom }}$ and clusters $\mathrm{Pt}_{10}, \mathrm{Pt}_{13}$ ) was adjusted to $1.0 \cdot 10^{14}$ atoms $\mathrm{cm}^{-2}$ which corresponds to $6.7 \%$ of a monolayer when compared to a monolayer of bulk platinum $\left(1.6 \cdot 10^{15}\right.$ atoms $\left.\mathrm{cm}^{-2}\right)$ [34]. The coverages determined based on the deposition currents show an error of approximately $20 \%$. A further description of the cluster selection and ion guiding can be found elsewhere [35].

For the electrodeposition of platinum nanoparticles of $\sim 230 \mathrm{~nm}$ in diameter $\left(\mathrm{Pt}_{\text {nano }}\right)$ a $2 \mathrm{mM}$ solution of potassium tetrachloroplatinate(II) $\left(\mathrm{K}_{2} \mathrm{PtCl}_{4}, 99 \%\right.$, Carbolution Chemicals) in $0.5 \mathrm{M}$ sulphuric acid (analytical grade, Carl Roth) as electrolyte was used. Before the cell was filled with the electrolyte, the cleaned titanium substrates with native titanium dioxide layer were sealed with a home build PTFE holder into a commercial three-electrode cell system PECC-2 from ZahnerElektrik GmbH \& Co. KG with $5.9 \mathrm{~mL}$ electrolyte volume. The effective deposition area on the substrate was $0.5 \mathrm{~cm}^{2}$. The counter electrode consisted of a platinum coil, whereas an $\mathrm{Ag} / \mathrm{AgCl}$ electrode as reference was used. The working electrode was connected by pressing a gold-plated electrode onto the titanium foil. During deposition the current density of $-0.5 \mathrm{~mA} \mathrm{~cm}{ }^{-2}$ was controlled by a Gamry (Interface 1000) potentiostat. After $2 \mathrm{~min}$ at ambient temperature and pressure, the precipitation was stopped. In order to remove electrolyte and other residues the deposited surface was rinsed with Millipore water (Merck Millipore, Milli-Q Reagent-Grade Water System). Afterwards, all samples were dried in a nitrogen stream and immediately brought in an ultra-high vacuum (UHV) chamber. Table 1 provides an overview of the samples. 
Tab. 1: Sample overview.

\begin{tabular}{llllrr}
\hline Sample & Support & Deposition type & Particle size & $\begin{array}{r}\text { Pt coverage/ } \\
\text { atoms } \mathbf{c m}^{-2}\end{array}$ & Pt mass $/ \boldsymbol{\mu g ^ { - 1 }}$ \\
\hline $\mathrm{TiO}_{2}$ & $\mathrm{TiO}_{2}$ & None & & & \\
$\mathrm{Pt}_{\text {atom }}$ & $\mathrm{TiO}_{2}$ & Magnetron sputtering & 1 atom & $1.0 \cdot 10^{14}$ & 0.03 \\
$\mathrm{Pt}_{10}$ & $\mathrm{TiO}_{2}$ & Magnetron sputtering & 10 atoms & $1.0 \cdot 10^{14}$ & 0.03 \\
$\mathrm{Pt}_{13}$ & $\mathrm{TiO}_{2}$ & Magnetron sputtering & 13 atoms & $1.0 \cdot 10^{14}$ & 0.03 \\
$\mathrm{Pt}_{\text {nano }}$ & $\mathrm{TiO}_{2}$ & Electrodeposition & $\sim 230 \mathrm{~nm}$ & $5.2 \cdot 10^{16} /$ & $16.9 / 0.10 *$ \\
& & & Bulk & $3.1 \cdot 10^{14} *$ & $0.52 *$ \\
$\mathrm{Pt}_{\text {bulk }}$ & $\mathrm{Pt}$ & None & $1.6 \cdot 10^{15} *$ & \\
\hline
\end{tabular}

*Pt coverage and Pt mass under consideration of surface atoms only.

\subsection{Characterisation}

Atomic force microscopy (AFM) and transmission electron microscopy (TEM) were applied to investigate the roughness and topology of the surfaces, as well as the size of the deposited clusters and nanoparticles. For AFM measurements a commercial benchtop microscope Nanosurf EasyScan 2 operated under ambient conditions with a vibration-free mounted table was used. The images were analysed with the open source software Gwyddion v2.53. High-resolution TEM images were taken in cooperation with the Jooß group from Göttingen (Germany) on a research device. All images were taken using the secondary electron (SE) mode at a potential of $10 \mathrm{kV}$ with an Everhart-Thornley detector for the overview images and a through-lens detector for the zoomed-in images.

Photoelectron spectroscopy measurements (XPS and UPS) took place within the integrated UHV laboratory DAISY-FUN within the Jaegermann group in Darmstadt (Germany). For XPS a high-resolution monochromatic Al K $\alpha$ X-ray source at $1486.74 \mathrm{eV}$ (XR 50, Specs Surface Nano Analysis GmbH) was used. The spectrometer was calibrated to the core levels of clean coinage metals (copper, silver, gold) as well as to the Fermi edge of silver. To estimate the energy resolution the full width at half maximum (FWHM) of the Au $4 \mathrm{f}_{7 / 2}$ core line was investigated showing a FWHM of $0.5 \mathrm{eV}$. The analyser was a Specs Surface Nano Analysis GmbH Phoibos 150 hemispherical analyser.

The electrochemical measurements took place in an identical setup to the electrodeposition of platinum nanoparticles using the Gamry (Interface 1000) potentiostat (see Chapter 2.1.1). The setup was operated in a three-electrode configuration. An aqueous solution of $0.1 \mathrm{M}$ sulphuric acid (analytical grade, Carl Roth) was the electrolyte for all electrochemical experiments. For cyclic voltammetry measurements, a scan rate of $10 \mathrm{mV} \mathrm{s}^{-1}$ was applied. 


\section{Results}

\subsection{Imaging}

Surface roughness has a direct influence on the total number of platinum atoms reachable by the solvent in electrochemistry and hence on the overall catalytic performance. Thus, before a well-founded insight into the catalytic activity is possible, a picture of the topologies of the surfaces must first be obtained. AFM was implemented, to investigate the surface of the titanium foils and platinum reference. Since both metals were produced by a similar rolling process, the topologies differ insignificantly. In Figure 1 the topology of the platinum reference is shown in a $56 \mu \mathrm{m}^{2}$ large image section. For all samples, the mean roughness lies within the 1-2 $\mathrm{nm}$ range with a roughness factor of $1.04 \pm 0.02$. This implies a very planar surface with only small deviations. Therefore, the effective surface area deviates only a few percents from the geometric area.

In addition to the topology of the blank support material, it is also necessary to gain information about the deposited particles. In order to achieve relevant data for the catalytic activity, a homogeneous coverage of isolated particles is needed. To verify this, additional samples were prepared on TEM grids for both deposition methods (magnetron sputtering and electrodeposition). A Matlab (R2016a) script was used to analyse the images obtained. To determine the size distribution and coverage, a floating background was subtracted first before converting the images from grey scale to binary, which allows a more simplified identification and analysis of individual particles. In order to obtain

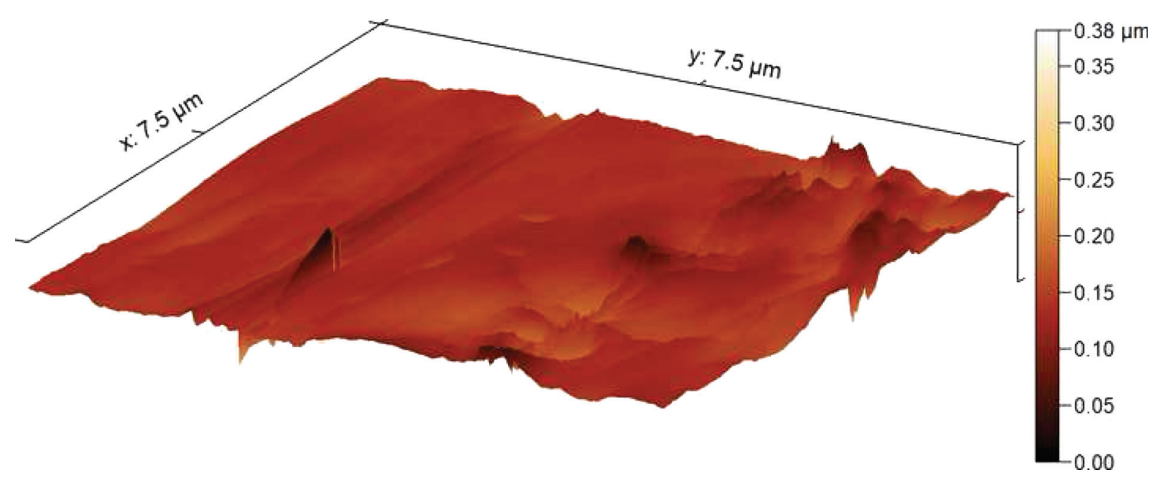

Fig. 1: AFM image of the bulk platinum reference sample $\mathrm{Pt}_{\text {bulk }}$. The colour bar indicates changes in the height of the sample (z-direction). 
a statistically meaningful quantity of particles for both the cluster/particle size and the total coverage, two different image sections were examined per sample. A larger section for determining the total coverage and one section with greater magnification for determining the size distribution based on the measurement of individual particles. The corresponding TEM images can be seen in Figure 2. Figure 2a illustrates $\mathrm{Pt}_{16}$-clusters deposited on a carbon TEM-grid, which was prepared by sputtering in analogy to the samples $\mathrm{Pt}_{\text {atom }}, \mathrm{Pt}_{10}$ and $\mathrm{Pt}_{13}$. The slightly larger $\mathrm{Pt}_{16}$-clusters were used to achieve better contrast, since larger particles are easier to identify within the TEM images. The obtained atomic coverage is in agreement with the coverage received from ion-current measurement during deposition within 20-40\%. Figure $2 \mathrm{~b}$ illustrates platinum nanoparticles deposited by electrodeposition on a $\mathrm{TiO}_{2}$ TEM-grid synthesised under the same conditions like the $\mathrm{Pt}_{\text {nano }}$ samples. Whereas the $\mathrm{Pt}_{16}$-clusters have a diameter of $(0.9 \pm 0.3) \mathrm{nm}$, the nanoparticles have an average diameter of $(232 \pm 90) \mathrm{nm}$. By TEM imaging of a sample cross-section, it became visible that the nanoparticles possess a half-sphere shape. Applying the spherical cluster approximation (SCA) [36] to the Pt atoms within the nanoparticles as hard spheres with a Wigner-Seitz radius of $136 \mathrm{pm}$ (calculated from the density of bulk platinum) [34], an average number of $3 \cdot 10^{8}$ atoms per nanoparticle is obtained. The number of Pt atoms on the surface adds up to $0.6 \%$ of the total atom number per nanoparticle.
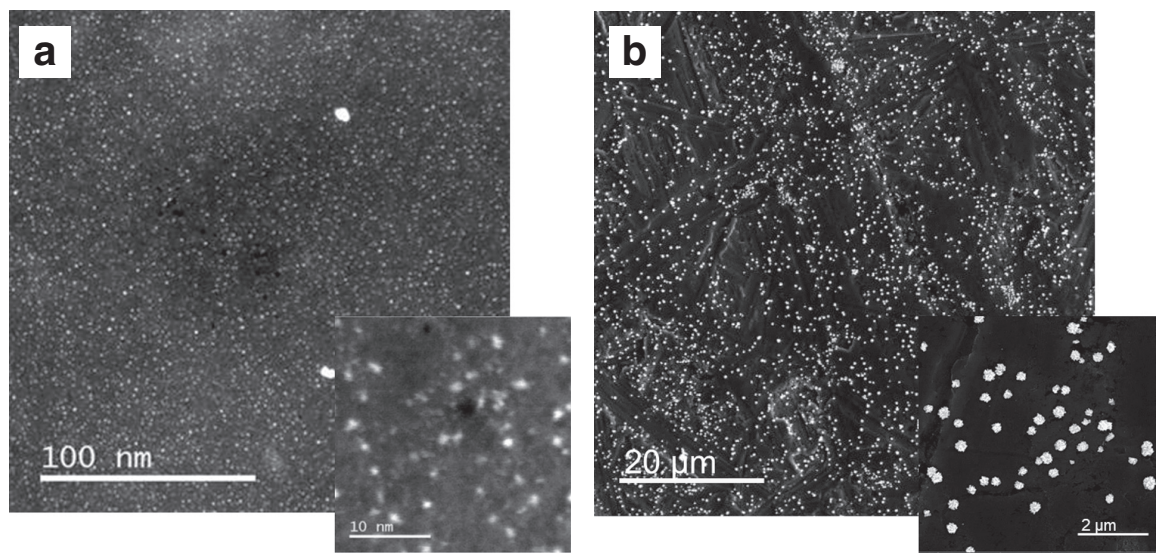

Fig. 2: TEM images of differently deposited Pt particles, overview and zoomed in section.

(a) Magnetron sputtered Pt clusters with a diameter $(0.9 \pm 0.3) \mathrm{nm}$ on a carbon TEM-grid.

(b) Electrodeposited Pt nanoparticles with an average diameter of (232 \pm 90$) \mathrm{nm}$ on a $\mathrm{TiO}_{2}$ TEM-grid. 


\subsection{Photoelectron spectroscopy}

After preparation, all samples were introduced directly into the integrated UHV system connected to the analysis chamber in which XPS and UPS measurements were performed in sequence. Firstly all samples were checked for unwanted contaminations other than residual carbon which could not be washed completely of previously or which readsorbed under ambient conditions during transfer into the vacuum system. In addition, the intensity of the Ti- and Pt-core lines were utilised to validate the Pt coverage obtained by the ion-current measurements during cluster deposition via magnetron sputtering. In order to calculate the coverage the dampening of the Ti2p intensity assuming a homogeneous layer of platinum was used. Two inelastic mean free paths (IMFPs) are needed for the calculation: Ti2p electrons in platinum from the NIST IMFP Database $(\lambda=13.34 \AA$ ) [37] and Ti2p electrons in $\mathrm{TiO}_{2}$. Due to a missing experimental value for the latter, the TPP$2 \mathrm{M}$ algorithm within the NIST IMFP Database for inorganic compounds was used with the following data for titanium dioxide: the number of valence electrons per molecule $=16$, band-gap for native titanium oxide $=3.05 \mathrm{eV}$ and a density of $4.22 \mathrm{~g} \mathrm{~cm}^{-3}$ [38] resulting in an IMFP of $\lambda=27.7 \AA$. This approach has a high amount of uncertainty leading to an error margin of approximately a factor of two. But this is still a feasible tool to re-validate the overall particle coverages. All values of the coverages determined from the current measurements are within this margin.

A comparison of the $\mathrm{Pt}_{4} \mathrm{f}_{7 / 2}$ core-levels reveals distinct electronic differences between the samples. For $\mathrm{Pt}_{\text {atom }}$ the core level is $72.9 \mathrm{eV}, 72.3 \mathrm{eV}$ for $\mathrm{Pt}_{10}$ and decreasing further to $72.1 \mathrm{eV}$ for $\mathrm{Pt}_{13}, 71.3 \mathrm{eV}$ for $\mathrm{Pt}_{\text {nano }}$ and $71.2 \mathrm{eV}$ for $\mathrm{Pt}_{\text {bulk }}$ as seen in Figure 3. In the case of the latter two samples, the usual line-shape for metallic core-lines can also be detected, which was not observed for the deposited clusters. The result for the $\mathrm{Pt}_{\text {nano }}$ sample is in agreement with platinum nanoparticles of comparable size on amorphous titan dioxide films [39, 40]. Compared to results from references [41, 42], the Pt4f $\mathrm{f}_{7 / 2}$ core-levels of the small cluster species $\left(\mathrm{Pt}_{\text {atom }}, \mathrm{Pt}_{10}, \mathrm{Pt}_{13}\right)$ are increased by $0.5-0.8 \mathrm{eV}$. This discrepancy can be explained by different $\mathrm{TiO}_{2}$ support effects in the case of crystalline oxide. The core-level shifts are due to modified final state screening precipitated by titania's electrophilicity and by particle size effects. Whereas electrons in metallic platinum easily screen a core hole created by the photoelectric effect and therefore stabilising the final state, this does not hold for very small particles. The final state energy is shifting due to a localised charge which is proportional to the inverse particle radius. Also, a partial polarisation of the valence electrons occurs since there are no delocalised conduction band electrons available [43-46]. Complementary the electronic structure of the support material is relevant for both the initial and the 


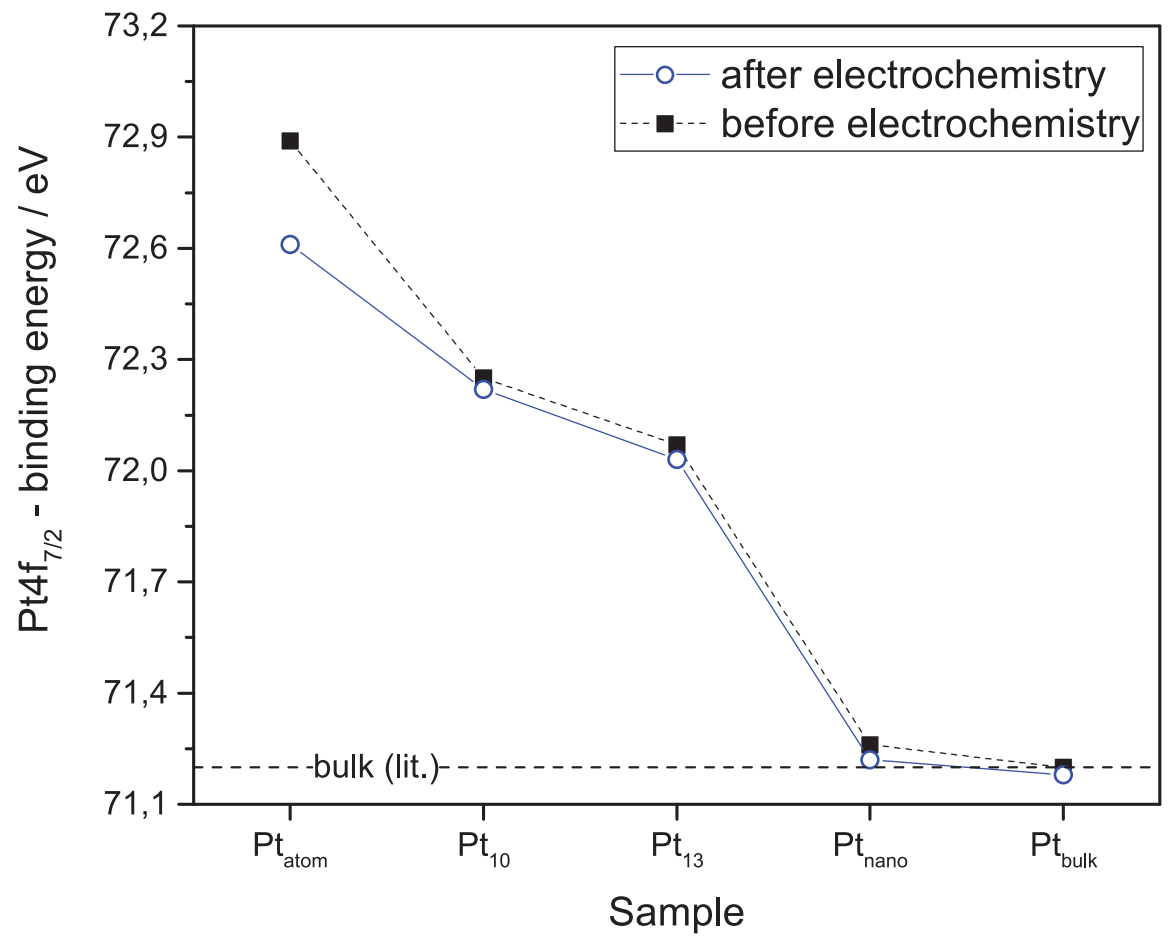

Fig. 3: $\mathrm{Pt}_{4 \mathrm{f}} \mathrm{f} / 2$ binding energies for different platinum species on native $\mathrm{TiO}_{2}$ before and after electrochemical measurements. The dashed line indicates the core-level for bulk platinum as known from literature [48].

final state effect. For titania-supported platinum clusters, this leads to a small positive charging of the cluster due to the high electronegativity of the oxygen in titania in comparison to platinum [26, 47]. Even though titanium is less electronegative, the resulting total charge will still be positive over the whole cluster (e.g. +0.97 e for $\mathrm{Pt}_{13}$ according to Wang et al. [26]). A more conductive support can compensate this effect up to a specific margin. Also, the charge carrier density, acidity and defect concentration have an impotent impact on the initial state effect and therefore on the core shift in comparison to the particle bulk material $[48,49]$. Overall a strong influence of cluster size onto the electronic structure can be seen.

A change in geometric and electronic structure for clusters and nanoparticles, in particular, precipitated though electrochemical measurements are well known from literature [50-54]. Which mechanism (agglomeration, migration, Oswald ripening, dissolving, detachment) is dominant depends strongly on the particle substrate combination [50-54]. As stated in Figure 3 for the majority of samples 
only small shifts in binding energy for the $\mathrm{Pt}_{4 \mathrm{f}} \mathrm{f}_{72}$ core-levels to lower values can be observed after electrochemical treatment indicating only minor modifications of the overall electronic structure of the platinum particles. This is not the case for platinum atoms supported on titania $\left(\mathrm{Pt}_{\text {atom }}\right)$, in as much as a clear shift of the binding energy to lower values can be recorded. The initial value for the Pt4f core-line of $72.9 \mathrm{eV}$ shifts to $72.6 \mathrm{eV}$ implying the formation of small clusters through sintering during the $\mathrm{CV}$ measurements. In contrast to larger particles, the platinum atom has much lower adsorption energy and a fewer number of possible anchoring sites, resulting in higher mobility on the surface. The platinum atom seems to agglomerate to dimers and small clusters. This matches with the obtained Pt $4 \mathrm{f}$ binding energy of $72.6 \mathrm{eV}$ which fits within the gap of Pt $\mathrm{Atom}_{\text {and }}$ $\mathrm{Pt}_{10}$. In addition, the total intensity of the platinum core-lines for all samples is altered through the electrochemical treatment resulting in a loss of 30-60\% after $5 \mathrm{~h}$ of measurement within the electrolyte. The samples $\mathrm{Pt}_{10}, \mathrm{Pt}_{13}$ and $\mathrm{Pt}_{\text {nano }}$ lose material from the surface rather than to change their structure due to ripening mechanisms. Other core-lines were not subject to major change apart from expected small quantities of remaining $-\mathrm{OH}$ groups.

Valence band spectra reveal additional information of the electronic structure of the catalytic systems near the Fermi-edge. Relating to Figure 4 between $4 \mathrm{eV}$ and $8 \mathrm{eV}$ a superposition of metal (Ti, $\mathrm{Pt}$ ) and oxide valence states can be found. In the UPS analysis of the plain native $\mathrm{TiO}_{2}$ surface the valence band edge appears at 3.1-3.5 eV, which is in good agreement with values from the literature $[55,56]$. The small Pt-species $\mathrm{Pt}_{a t o m}, \mathrm{Pt}_{10}$ and $\mathrm{Pt}_{13}$ did not reach a metallic state, regarding the lack of density of states (DOS) near the Fermi-level. In contrast, $\mathrm{Pt}_{\text {nano }}$ and $\mathrm{Pt}_{\text {bulk }}$ show a high intensity of DOS at the Fermi-level indicating metallic behaviour matching with the value of Pt $4 \mathrm{f}$ core-lines for very large particles and bulk platinum. Density functional theory calculations from Wang et al. showed that the DOS in the valence gap of $\mathrm{TiO}_{2}$ is filled by deposited $\mathrm{Pt}_{\mathrm{n}}$-clusters with $n=5,8,13,19$ [26]. The resulting valence band gap narrows with an increase in cluster size analogous to the experimental data obtained here. In the UPS spectra of the platinum deposited surfaces the band edge can be found at 1.5-1.9 eV for $\mathrm{Pt}_{\text {atom }}, 0.7-0.8 \mathrm{eV}$ for $\mathrm{Pt}_{10}$ and $0.25-0.4 \mathrm{eV}$ for $\mathrm{Pt}_{13}$. This is in good agreement with calculations from Wang et al. who received $3.5 \mathrm{eV}$ for pure titania, $0.87 \mathrm{eV}$ for $\mathrm{Pt}_{8}$ and $0.45 \mathrm{eV}$ for $\mathrm{Pt}_{13}$ supported on $\mathrm{TiO}_{2}(101)$ [26].

Valence electrons near the Fermi level as well as the highest occupied molecular orbital (HOMO) and lowest unoccupied molecular orbital (LUMO) positions are of decisive importance for the activity in catalysis [57]. According to Figure 4 at least the position of the HOMO can be tuned by optimising the cluster size. Further information regarding the LUMO could be gathered employing i.e. inverse photoemission spectroscopy. According to size-dependent shifts of the unoccupied 


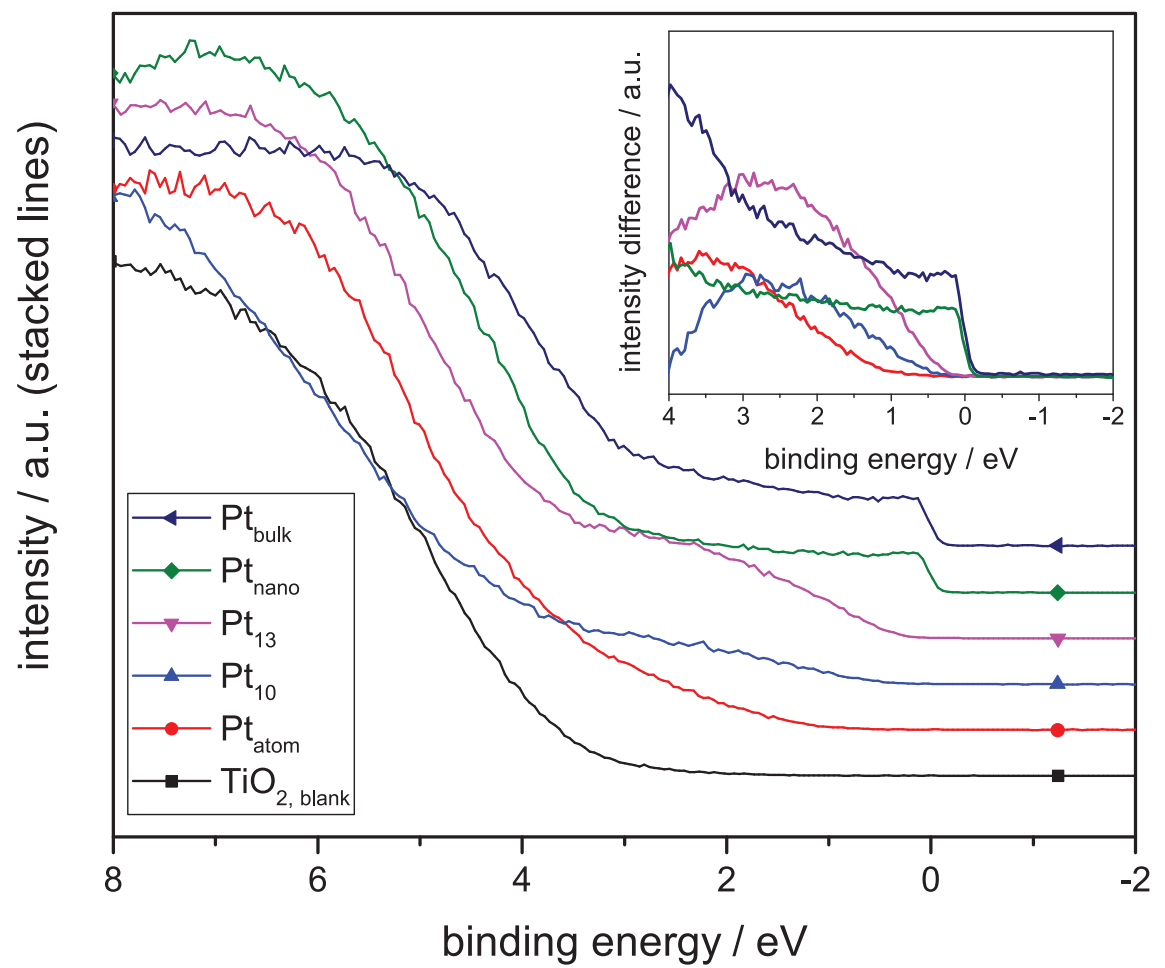

Fig. 4: UPS valence band spectra for different platinum catalysts on native $\mathrm{TiO}_{2}$. The energy of excitation was $\mathrm{h} v=40.81 \mathrm{eV}$ (Helium II). For reasons of clarity the spectra are stacked, all lines below $-2 \mathrm{eV}$ are at zero intensity. The inserted graph shows the difference-spectra of the platinum catalysts (as-is spectra subtracted by the $\mathrm{TiO}_{2}$ spectrum). Because the total amount of platinum is varying for different samples, the intensities of the measured photoelectrons of the various spectra should not be compared to each other.

states for small Pt-clusters can be found in literature [58]. To reduce hydrogen, an electron has to go through a two-step process (if not concerted) in which it passes from the substrate (electrode) to the cluster and finally to the hydrogen. Both processes require different optimal LUMO positions. The LUMO of the cluster must be located above the reduction potential of hydrogen, otherwise hydrogen can not be reduced. But to maximise the lifetime of an excited state with the electron located on the cluster, the electron needs to be trapped there. This can be realised with a low position of the LUMO beneath the conduction band of the supporting material. In the future, it might be interesting to investigate also the DOS of the unoccupied states for supported clusters. But the here presented data clearly suggest that the valence orbitals could be tuned by changing the particle size of the catalytic active cluster [59]. 


\subsection{Electrochemistry}

To ensure proper functionality of the used electrochemical setup and to gain a benchmark, two reference systems are studied, i.e. a platinum foil $\mathrm{Pt}_{\text {bulk }}$ and clean $\mathrm{TiO}_{2}$. They are also important for comparison with the deposited samples, as well as with data from the literature. All cyclic voltammetry (CV) measurements are taken after a set of measurements to make sure, that quasi-stationary conditions were reached. The set consisted of impedance measurements between 0.1 $\mathrm{Hz}$ and $100 \mathrm{kHz}$ ranging from 0.0 to $-0.1 \mathrm{~V}$ vs RHE (reference hydrogen electrode) with 10 points per decade, cyclic voltammetry from +0.5 to $-0.6 \mathrm{~V}$ vs RHE with $50 \mathrm{mV} / \mathrm{s}(15 \times)$ and with $10 \mathrm{mV} / \mathrm{s}(10 \times)$. In between the individual measurements, the open-circuit potential was held for $300 \mathrm{~s}$ each. In total each sample was in contact with the electrolyte for about $5 \mathrm{~h}$. The measurements are done in $0.1 \mathrm{M}$ $\mathrm{H}_{2} \mathrm{SO}_{4}$ with a scan rate of $10 \mathrm{mV} \mathrm{s}{ }^{-1}$. Each cycle starts at an electric potential of $+0.5 \mathrm{~V}$ vs the value of the reference hydrogen electrode moving on to the turning point of $-0.6 \mathrm{~V}$ vs RHE and back to $+0.5 \mathrm{~V}$ vs RHE. As shown in Figure 5, the blank $\mathrm{TiO}_{2}$ reaches a maximum of $-1.6 \mathrm{~mA} \mathrm{~cm}{ }^{-2}$ at $-0.6 \mathrm{~V}$ vs RHE and is therefore nearly inactive for the HER, which is in good agreement with the data from Proch et al. (under $2 \mathrm{~mA} \mathrm{~cm}{ }^{-2}$ in $0.1 \mathrm{M} \mathrm{HClO}_{4}$, identical scan rate) [33]. The feature within the current densities slightly above $0.1 \mathrm{~V}$ vs RHE indicates the desorption of weekly bound hydrogen onto the electrode surface [60]. The negative branch below $0 \mathrm{~V}$ vs RHE is appertaining to the hydrogen evolution reaction. The hysterical course in this region is typical evidence for hydrogen bonding onto the platinum surface [61, 62]. Those features are more distinct for the $\mathrm{Pt}_{\text {bulk }}$ and $\mathrm{Pt}_{\text {nano }}$
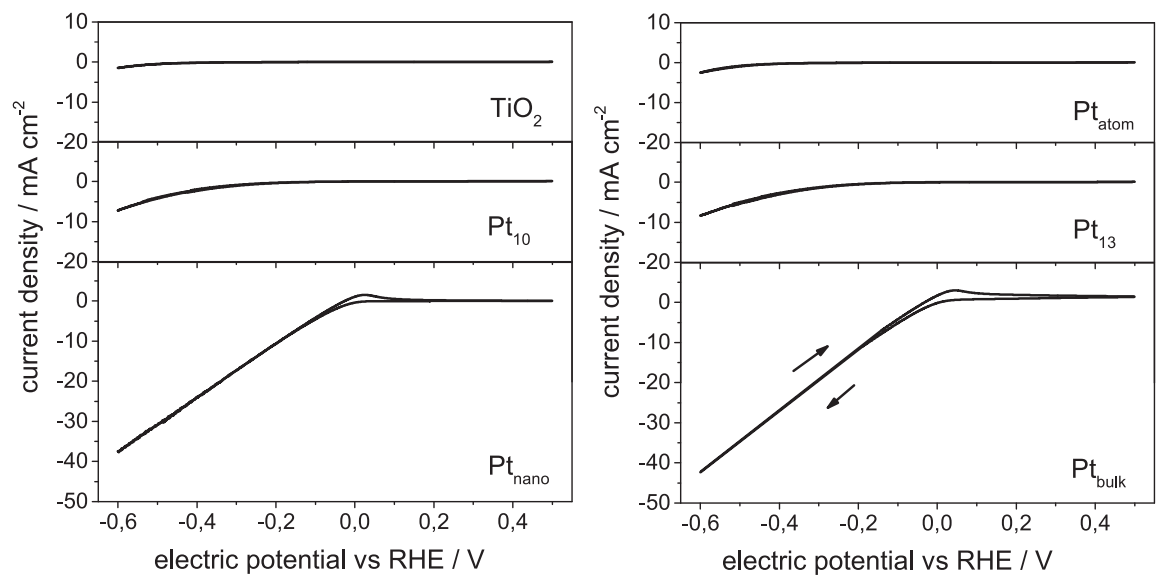

Fig. 5: $\mathrm{CVs}$ of different platinum catalysts on $\mathrm{TiO}_{2}$ support compared to bulk platinum in $0.1 \mathrm{M}$ $\mathrm{H}_{2} \mathrm{SO}_{4}$ acquired with a scan rate of $10 \mathrm{mV} \mathrm{s}^{-1}$. 
samples rather than for the cluster deposited surfaces. The sample $\mathrm{Pt}_{\text {atom }}$ shows only a slight increase in catalytic performance compared with the $\mathrm{TiO}_{2}$-reference. If the used catalyst lowers the overpotential far enough as seen for the $\mathrm{Pt}_{\text {bulk }}$ reference, significant current densities of over $40 \mathrm{~mA} \mathrm{~cm}^{-2}$ can be reached. This is also the case for the deposited platinum particles $\mathrm{Pt}_{\text {nano }}$, where nearly identical values to the bulk material are obtained.

Considering the high current densities achieved with $\mathrm{TiO}_{2}$ as support material, problems with electric resistance due to the isolating properties of the native oxide on the titanium foil can be ruled out. In addition to electron tunnelling through the thin oxide layer, electron transport can also occur through the bulk titania which is mainly determined by a strong electron-phonon coupling $[63,64]$. Due to the thickness of the native oxide layer of about $2.5 \mathrm{~nm}$ and an overall electric conductivity of the $\mathrm{TiO}_{2}$ in the range of $10^{-3}(\Omega \mathrm{m})^{-1}-10^{0}(\Omega \mathrm{m})^{-1}[32,65]$, the resulting resistance of the native oxide layer is in the range of the detection limit of about $1 \mathrm{~m} \Omega$. Hence the electron transport can be disregarded and only the electrocatalytic activity is investigated for our experiments. When discussing the catalytic performance the current density value for the titanium dioxide as a reference system was subtracted for further current densities, so only the additional platinum-induced catalytic activity is taken into account.

The wide range of catalytic species, as well as the large gap of overall used catalytic material, leads to difficulties in the comparability of catalytic activity. The usual comparison of the considered catalysts by the selection of a current density (e.g. $-1.0 \mathrm{~mA} \mathrm{~cm}^{-1}$ ) to be reached and the consideration of the corresponding electric potential is not suitable in this case. The so gained potentials could only be analysed for the as-is samples but cannot be put in relation to the used catalyst amount which is necessary here. The catalytic activity needs to be evaluated as a current density at a potential of choice. The used CVs are not IR-corrected in addition due to the variation in the onset potentials for the HER. With an IRcorrection the lowest potentials for the bulk platinum would be about $-0.15 \mathrm{~V}$ vs RHE which is still below the onset potentials for the small cluster species at this low cluster coverage. The catalytic activity will therefore depend on the potential and coverage choosen. The different CVs can still be compared since the sum of resistances (electrolyte, cables, support) is equal for all investigated samples ( $22 \pm 0.5 \Omega$, determined by impedance measurement). This resistance is also the reason why a linear increase can be found for high current densities rather than an exponential one.

The current densities at $-0.6 \mathrm{~V}$ vs RHE for the supported monodisperse platinum species $\left(\mathrm{TiO}_{2}\right.$ reference subtracted) with $-1.18 \mathrm{~mA} \mathrm{~cm}{ }^{-1}$ for $\mathrm{Pt}_{\text {atom }}$, $-5.56 \mathrm{~mA} \mathrm{~cm}^{-1}$ for $\mathrm{Pt}_{10}$ and $-6.86 \mathrm{~mA} \mathrm{~cm}^{-1}$ for $\mathrm{Pt}_{13}$ are still low compared to the value of the large nanoparticles or even to the bulk material. However, these 
values are obtained using a different amount of platinum and can hardly be compared with each other by considering the current densities alone. To gain a better inside, the total mass of platinum was determined for all samples using the coverages (see Table 1) and set in relation to the current densities. The results can be seen in Figure 6 where the current densities at $-0.6 \mathrm{~V}$ vs RHE are compared with the mass-specific current densities for all samples. In summary, much

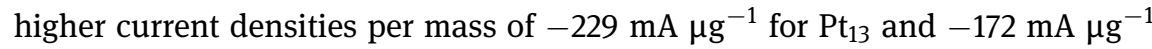
for $\mathrm{Pt}_{10}$ are achieved for the clusters, than for the other deposited samples $\left(\mathrm{Pt}_{\text {atom }}\right.$ : $-36 \mathrm{~mA} \mu \mathrm{g}^{-1}, \mathrm{Pt}_{\text {nano }}$ : $-1.16 \mathrm{~mA} \mu \mathrm{g}^{-1}$ ).

In terms of activity per mass, $\mathrm{Pt}_{\text {nano }}$ and $\mathrm{Pt}_{\text {bulk }}$ show a low specific catalytic activity due to the unfavourable surface-to-volume ratio. To allow a correlation with these samples as well, only the surface atoms can be used. Specifically, this corresponds to the first atomic layer for $\mathrm{Pt}_{b u l k}$ and the outer most layer for the Pt-

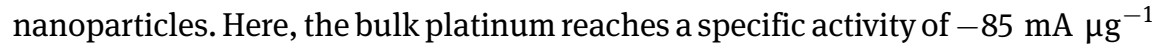

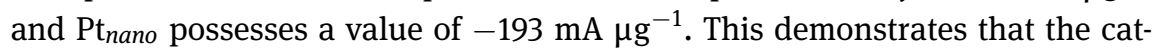
alytic activity varies only by about $20 \%$ considering Pt clusters with 10 atoms and nanoparticles with $10^{8}$ atoms when taking the number of surface atoms into

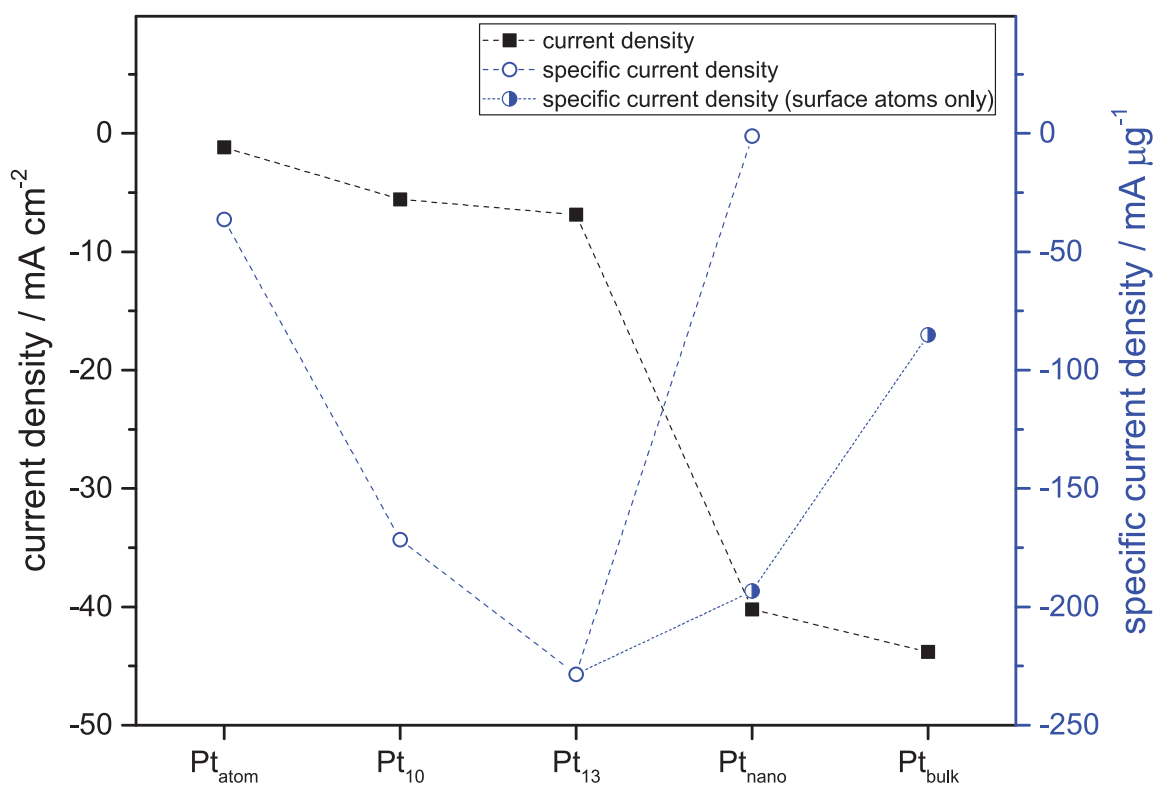

Sample

Fig. 6: Current densities and mass-specific current densities at $-0.6 \mathrm{~V}$ vs RHE in $0.1 \mathrm{M} \mathrm{H}_{2} \mathrm{SO}_{4}$ for different platinum catalysts on $\mathrm{TiO}_{2}$-support compared to bulk platinum. For $\mathrm{Pt}_{\text {nano }}$ and $\mathrm{Pt}_{\text {bulk }}$ the specific current densities for the surface atoms only are shown in addition. 


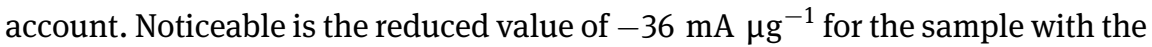
least efficient electrocatalyst $\mathrm{Pt}_{\text {atom }}$. If this is caused by an unideal LUMO position or by a missing second-layer of platinum, which is needed to achieve a proper catalytic activity for titania-supported platinum species, as [26] is still an open question.

If the catalytic activities are directly compared with the Pt4f core levels, no clear trend can be found. This is also the case for the valence band spectra and HOMO positions. However, Weber et al. were able to achieve a direct correlation of catalytic activity with core level position for small platinum clusters for ethanol electro-oxidation on indium tin oxide (ITO) [66] or the oxygen evolution reaction on ITO and glassy carbon [67]. Nevertheless, the same clusters on ITO used for oxygen reduction do not follow a trend regarding the Pt4f core-levels [13]. Effects induced due to changes in the valence electronic structure might be of minor importance compared to the influence of reachability and the overall amount of active sites. Still, there might be an optimum for both in the case of $\mathrm{Pt}_{10}$ and $\mathrm{Pt}_{13}$, but to clarify this more experiments with a wider range of cluster sizes are required. Here, the best activity can be seen for the subnanometer-clusters. Taking the total amount of platinum into account this is even much more pronounced. If only the surface atoms are considered the catalytic activity for the platinum clusters and the larger nanoparticles lies within $20 \%$.

A high reactivity with ambient oxygen can also have altered the activity since for all samples the measurements took place under application-related ambient conditions. The particles may have an even higher catalytic activity without the presence of residual gases, as already known from the literature [66, 68]. The active site tends to be the (partially) oxidised platinum on the oxide to cluster boundary [69], creating an increase in activity for the $\mathrm{H}-\mathrm{H}$ coupling on the surface, especially for small Pt-clusters. An activation with elemental oxygen forming PtOx is not possible and would even result in a loss of catalytic performance $[66,68]$. The activation effect drops off quickly for additional layers of platinum atoms and hence diminishing the activity for large clusters [70]. This predicts the highest activity per atom to be found for clusters without core atoms [71] which is also observed here for Pt nanoclusters having a size of about 10 atoms.

In agreement with the results from Proch et al. [68] after the electrochemical measurements, some degradation of the platinum catalysts, especially for the small clusters were found. These could be seen as a Pt4f core line intensity loss as well as shifts to lower binding energies in the case of $\mathrm{Pt}_{\text {atom }}$ after hours within the electrochemical setup, mostly induced through detachment and in the case of $\mathrm{Pt}_{\text {atom }}$ additional agglomeration took place. Since the positions of Pt4f core lines are strongly dependent on the cluster size, they give a good insight into the change of the size during the electrochemical measurements. For all investigated 
platinum catalysts, besides of the supported platinum atoms, no Pt4f shift could be observed. This is in good agreement with results from the literature [72] were a dissolution of platinum and therefore a reduction in particle size was found at potentials below $-0.9 \mathrm{~V}$ vs RHE and could not be observed at $-0.6 \mathrm{~V}$ vs RHE as used in this work. As described before, the increase in cluster size, which causes the core level shifting towards lower binding energies for the platinum atoms, can be explained due to the high mobility of single atoms resulting in agglomeration to slightly larger species on the titania surface [73]. After the set of electrochemical measurements, about $5 \mathrm{~h}$ in total, up to $60 \%$ of Pt4f intensity loss was observed for all deposited particles $\left(\mathrm{Pt}_{\text {atom }}, \mathrm{Pt}_{10}, \mathrm{Pt}_{13}\right.$ and $\mathrm{Pt}_{\text {nano }}$ ) independent of size and deposition technique. The soft-landing regime ensures the integrity of cluster geometry, but results in a much smaller particle-support interaction and binding energy than with embedded or even implanted particles [74]. Since the particles are only soft-landed on the $\mathrm{TiO}_{2}$ substrate and not further embedded into the surface they can be detached easily if in contact with a liquid electrolyte.

\section{Conclusion}

We were able to investigate the influence of system size of platinum catalysts for the HER. By applying different deposition techniques, it was possible to investigate a variety of catalytic species reaching from bulk platinum down to the study of isolated deposited platinum atoms. Strong size dependence of the electronic structure was found when taking the core level binding energies as well as the valance band structure into account. All investigated platinum catalysts are able to catalyse the HER. Especially the small monodisperse deposited clusters $\mathrm{Pt}_{10}$ and $\mathrm{Pt}_{13}$ were able to outperform bulk platinum, which is already known as good HER catalyst, in terms of activity per mass. In contrast to nanoclusters with about ten atoms, isolated Pt-atoms show a significantly reduced catalytic activity.

Acknowledgement: We like to thank the SPP 1613 priority program from the Deutsche Forschungs Gesellschaft DGF gratefully for funding (Funder Id: http://dx.doi.org/10.13039/501100001659, project SCHA 885/13-2). We also thank the group of Prof. Jooß in Göttingen (Germany) for the TEM measurements. Julian Baranyai thanks the Fond der Chemischen Industrie and Torben Schmidt thanks the Deutsche Bundesstiftung Umwelt for financial support.

\section{References}

1. P. V. Kamat, J. Phys. Chem. B 106 (2002) 7729. 
2. F. Zaera, Chem. Soc. Rev. 42 (2013) 2746.

3. Y. Xia, H. Yang, C. T. Campbell, Acc. Chem. Res. 46 (2013) 1671.

4. M. Ge, J. Cai, J. locozzia, C. Cao, J. Huang, X. Zhang, J. Shen, S. Wang, S. Zhang, K.-Q. Zhang, Y. Lai, Z. Lin, Int. J. Hydrog. Energy 42 (2017) 8418.

5. L. Liu, A. Corma, Chem. Rev. 118 (2018) 4981.

6. T. Kitanosono, K. Masuda, P. Xu, S. Kobayashi, Chem. Rev. 118 (2018) 679.

7. S. E. F. Kleijn, S. C. S. Lai, M. T. M. Koper, P. R. Unwin, Angew. Chem. Int. Ed. Engl. 53 (2014) 3558.

8. E. C. Tyo, S. Vajda, Nat. Nanotechnol. 10 (2015) 577.

9. M. Rondelli, G. Zwaschka, M. Krause, M. D. Rötzer, M. N. Hedhili, M. P. Högerl, V. D’Elia, F. F. Schweinberger, J.-M. Basset, U. Heiz, ACS Catal. 7 (2017) 4152.

10. Y. Watanabe, Sci. Technol. Adv. Mat. 15 (2014) 063501.

11. S. Vajda, M. G. White, ACS Catal. 5 (2015) 7152.

12. P. C. K. Vesborg and T. F. Jaramillo, RSC Adv. 2 (2012) 7933.

13. A. von Weber, E. T. Baxter, H. S. White, S. L. Anderson, J. Phys. Chem. C 119 (2015) 11160.

14. F. F. Schweinberger, M. J. Berr, M. Döblinger, C. Wolff, K. E. Sanwald, A. S. Crampton, C. J. Ridge, F. Jäckel, J. Feldmann, M. Tschurl. U. Heiz, J. Am. Chem. Soc. 135 (2013) 13262.

15. K. Welter, N. Hamzelui, V. Smirnov, J.-P. Becker, W. Jaegermann, F. Finger, J. Mat. Chem. A 6 (2018) 15968.

16. W. A. Smith, I. D. Sharp, N. C. Strandwitz, J. Bisquert, Energy Environ. Sci. 8 (2015) 2851.

17. J. Cui, T. Allen, Y. Wan, J. Mckeon, C. Samundsett, Di Yan, X. Zhang, Y. Cui, Y. Chen, P. Verlinden, A. Cuevas, Sol. Energy Mat. Sol. C. 158 (2016) 115.

18. T. C. Li, M. S. Góes, F. Fabregat-Santiago, J. Bisquert, P. R. Bueno, C. Prasittichai, J. T. Hupp, T. J. Marks, J. Phys. Chem. C 113 (2009) 18385.

19. P. Chen, X. Yin, M. Que, Y. Yang, W. Que, RSC Adv. 6 (2016) 57996.

20. S. Proch, K. Kodama, S. Yoshino, N. Takahashi, N. Kato, Y. Morimoto, Electrocatalysis 7 (2016) 362.

21. B. E. Hayden, D. Pletcher, J.-P. Suchsland, L. J. Williams, Phys. Chem. Chem. Phys. 11 (2009) 9141.

22. D. Schäfer, C. Mardare, A. Savan, M. D. Sanchez, B. Mei, W. Xia, M. Muhler, A. Ludwig, W. Schuhmann, Anal. Chem. 83 (2011) 1916.

23. B. E. Hayden, Acc. Chem. Res. 46 (2013) 1858.

24. K. Lee, A. Mazare, P. Schmuki, Chem. Rev. 114 (2014) 9385.

25. M. F. Lichterman, S. Hu, M. H. Richter, E. J. Crumlin, S. Axnanda, M. Favaro, W. Drisdell, Z. Hussain, T. Mayer, B. S. Brunschwig, N. S. Lewis, Z. Liu, H.-J. Lewerenz, Energy Environ. Sci. 8 (2015) 2409.

26. D. Wang, Z.-P. Liu, W.-M. Yang, ACS Catal. 8 (2018) 7270.

27. G. Hass, A. P. Bradford, J. Opt. Soc. Am. 47 (1957) 125.

28. J. Lausmaa, B. Kasemo, H. Mattsson, Appl. Surf. Sci. 44 (1990) 133.

29. E. McCafferty, J. P. Wightman, Surf. Interface Anal. 26 (1998) 549.

30. E. McCafferty, J. Wightman, Appl. Surf. Sci. 143 (1999) 92.

31. C. Sittig, M. Textor, N. D. Spencer, M. Wieland, P.-H. Vallotton, J. Mat. Sci. 10 (1999) 35.

32. C. J. Boxley, H. S. White, C. E. Gardner, J. V. Macpherson, J. Phys. Chem. B 107 (2003) 9677.

33. S. Proch, S. Yoshino, N. Takahashi, J. Seki, S. Kosaka, K. Kodama, Y. Morimoto, Electrocatalysis 9 (2018) 608.

34. P. R. Norton, J. A. Davies, T. E. Jackman, Surf. Sci. Lett. 122 (1982) L593. 
35. B. Elger, T. Schmidt, S. Krähling, F. Neuberger, R. Schäfer, Rev. Sci. Instrum. 88 (2017) 063303.

36. G. L. Hornyak, H. F. Tibbals, J. Dutta, J. J. Moore, Introduction to Nanoscience and Nanotechnology, CRC Press, Hoboken (2008).

37. C. J. Powell, A. Jablonski, NIST Electron Inelastic-Mean-Free- (2010).

38. D. C. Cronemeyer, Phys. Rev. 87 (1952) 876.

39. E. I. Vovk, A. V. Kalinkin, M. Y. Smirnov, I. O. Klembovskii, V. I. Bukhtiyarov, J. Phys. Chem. C 121 (2017) 17297.

40. Z. H. Al-Azri, W.-T. Chen, A. Chan, V. Jovic, T. Ina, H. Idriss, G. I. Waterhouse, J. Catal. 329 (2015) 355.

41. Y. Watanabe, N. Isomura, J. Vac. Sci. Technol. 27 (2009) 1153.

42. N. Isomura, X. Wu, H. Hirata, Y. Watanabe, J. Vac. Sci. Technol. 28 (2010) 1141.

43. V. D. Borman, M. A. Pushkin, V. N. Tronin, V. I. Troyan, J. Exp. Theor. Phys. 110 (2010) 1005.

44. D.-Q. Yang, E. Sacher, Appl. Surf. Sci. 195 (2002) 187.

45. F. S. Roberts, S. L. Anderson, A. C. Reber, S. N. Khanna, J. Phys. Chem. C 119 (2015) 6033.

46. W. Eberhardt, P. Fayet, D. M. Cox, Z. Fu, A. Kaldor, R. Sherwood, D. Sondericker, Phys. Scr. (1990) 892.

47. C. L. Muhich, Y. Zhou, A. M. Holder, A. W. Weimer, C. B. Musgrave, J. Phys. Chem. C 116 (2012) 10138

48. J. F. Moulder, W. F. Stickle, P. E. Sobol, K. D. Bomben, J. Chastain, R. C. King Jr. (Ed.), Handbook of X-ray Photoelectron Spectroscopy: A Reference Book of Standard Spectra for Identification and Interpretation of XPS Data, Physical Electronics, Eden Prairie, Minn. (1995).

49. A. S. Crampton, M. D. Rötzer, U. Landman, U. Heiz, ACS Catal. 7 (2017) 6738.

50. P. V. Dudin, P. R. Unwin, J. V. Macpherson, J. Phys. Chem. C 114 (2010) 13241.

51. P. L. Redmond, A. J. Hallock, L. E. Brus, Nano Lett. 5 (2005) 131.

52. H. L. Xin, J. A. Mundy, Z. Liu, R. Cabezas, R. Hovden, L. F. Kourkoutis, J. Zhang, N. P. Subramanian, R. Makharia, F. T. Wagner, D. A. Muller, Nano Lett. 12 (2012) 490.

53. Y. Yu, H. L. Xin, R. Hovden, D. Wang, E. D. Rus, J. A. Mundy, D. A. Muller, H. D. Abruña, Nano Lett. 12 (2012) 4417.

54. J. Quinson, M. Röefzaad, D. Deiana, T. W. Hansen, J. B. Wagner, M. Nesselberger, A. S. Crampton, C. J. Ridge, F. F. Schweinberger, U. Heiz, M. Arenz, Electrochim. Acta 277 (2018) 211.

55. Y. W. Chung, W. J. Lo, G. A. Somorjai, Surf. Sci. 64 (1977) 588.

56. K. D. Schierbaum, S. Fischer, M. C. Torquemada, J. L. de Segovia, E. Roman, Surf. Sci. 345 (1996) 261.

57. P. J. Fackler, Metal-Metal Bonds and Clusters in Chemistry and Catalysis, Springer, New York, NY (2013).

58. H. Tsunoyama, Y. Yamano, C. Zhang, M. Komori, T. Eguchi, A. Nakajima, Top. Catal. 61 (2018) 126.

59. B. von Issendorff and O. Cheshnovsky, Ann. Rev. Phys. Chem. 56 (2005) 549.

60. L. D. Burke, Platinum Metals Rev. 1994 (1994) 166.

61. S. Motoo, N. Furuya, J. Electroanal. Chem. 172 (1984).

62. J. Clavilier, R. Albalat, R. Gomez, J. M. Orts, J. M. Feliu, A. Aldaz, J. Electroanal. Chem. 330 (1992) 489.

63. E. Hendry, F. Wang, J. Shan, T. F. Heinz, M. Bonn, Phys. Rev. B 69 (2004) 347.

64. M. A. Henderson, Surf. Sci. Rep. 66 (2011) 185. 
65. A. Yildiz, S. B. Lisesivdin, M. Kasap, D. Mardare, J. Non-Cryst. Solids 354 (2008) 4944.

66. A. von Weber, E. T. Baxter, S. Proch, M. D. Kane, M. Rosenfelder, H. S. White, S. L. Anderson, Phys. Chem. Chem. Phys. 17 (2015) 17601.

67. A. von Weber, S. L. Anderson, Acc. Chem. Res. 49 (2016) 2632.

68. S. Proch, M. Wirth, H. S. White, S. L. Anderson, J. Am. Chem. Soc. 135 (2013) 3073.

69. J. Xing, H. B. Jiang, J. F. Chen, Y. H. Li, L. Wu, S. Yang, L. R. Zheng, H. F. Wang, P. Hu, H. J. Zhao, H. G. Yang, J. Mat. Chem. A 1 (2013) 15258.

70. H. Chen, P. Li, N. Umezawa, H. Abe, J. Ye, K. Shiraishi, A. Ohta, S. Miyazaki, J. Phys. Chem. C 120 (2016) 5549.

71. G.-F. Wei, Z.-P. Liu, Chem. Sci. 6 (2015) 1485.

72. S. Cherevko, N. Kulyk, K. J. Mayrhofer, Nano Energy 29 (2016) 275.

73. C.-T. Yang, N. Balakrishnan, V. R. Bhethanabotla, B. Joseph, J. Phys. Chem. C 118 (2014) 4702.

74. V. N. Popok, I. Barke, E. E. Campbell, K.-H. Meiwes-Broer, Surf. Sci. Rep. 66 (2011) 347. 